\title{
Der Mann mit der Kamera. Zur Kritik am dokumentarischen Realismus in Jean-Luc Godards Kurzfilm Caméra-œeil
}

\author{
Regine Prange
}

Aus dem Kaleidoskop der Ir überwiegend dokumentarischen Episoden des von Chris Marker editierten Kooperativ-Films Loin du Viet-nam $(1967),{ }^{2}$ welche das Geschehen in Vietnam, Protest- und Unterstützungsaktionen in den USA, historische Aufnahmen aus FranzösischIndochina und Streiks wie Protest im zeitgenössischen Frankreich zeigen, ragt Godards Beitrag Caméra-ail, der nach rund so Minuten Spieldauer eingefügt ist, heraus; nicht nur, weil er die Rolle des Intellektuellen bzw. des Künstlers im politischen Kampf problematisiert, also die Möglichkeit seiner Chance zum wirksamen Engagement hinterfragt. Diese Problematik thematisiert auch schon der lange, von dem Schauspieler Bernard Fresson vorgetragene Monolog des Schriftstellers Claude Ridder im Beitrag Alain Resnais', insofern er die innere Widersprüchlichkeit, ja die unwillentliche und unaufhebbare Verstricktheit der Protestbewegung in den von ihr bekämpften Herrschaftsapparat eindringlich beschreibt. ${ }^{3}$ Wie um diese

I Überarbeiteter Text eines Vortrags, der am Ir.6.2008 im Rahmen der vom Kunstgeschichtlichen Institut der Goethe-Universität initiierten Reihe ,Frankfurter filmhistorische Vorträge' zum Thema ,Kino $68^{4}$ im Deutschen Filmmuseum (DIF) gehalten wurde. Zum Ganzen des Films referierte Ralf M. Fischer. Ich zitiere in Anm. 3 aus seinem mir vorliegenden Manuskript. Zur Kommentierung des Filmganzen siehe auch Thomas Thode: Loin du Viêt-nam [Fern von Vietnam], 1967, in: Birgit Kämper und Thomas Thode (Hg.): Chris Marker. Filmessayist, München 1997, S. 251-255. Einer ähnlichen Thematik war mein Referat über ,Godard und die Selbstkritik des Kinos' für das von Gerhard Preyer initiierte Transdisziplinäre Gespräch der ProtoSociology am 10.7.2009 im Frankfurter Hilton gewidmet.

2 Regie führten außer Godard Alain Resnais, William Klein, Joris Ivens, Agnes Varda und Claude Lelouch.

3 "In an unfortunately theatrical tableau, Resnais', intellectual', for example, dissects his own guilt: he remembers his relieved affection for the bubblegum-chewing GI's of the second World War, speaks sardonically of the $40,000,000$ French anticolonialists, and characterizes Vietnam as the "first war everyone can see" (on television), and yet do nothing about. He ruminates on the ineffectiveness of, Gustave, a photo of a burned Japanes soldier, reprinted everywhere, which, in twenty years of standing for the bestiality of war, has done nothing." Max Kozloff: Shooting at Wars, in: Film Quarterly, Vol. 21, no.2, Winter 1967/68, S. 27-31, hier S. 30. Ralf M. Fischer kommentiert die Sequenz: „Claude Ridder soll für einen Filmproduzenten eine Rezension über Herman Kahns Buch ,On Escalation' [das von der Eskalationsleiter und vom Atomkrieg handelt] aus dem Jahr 1965 schreiben. Er räsoniert gegenüber seiner stillschweigenden Frau über frühere Kriege, die Rolle der Amerikaner als Befreier im Zweiten Weltkrieg und über die Bedeutung von Vietnam. Dabei wägt er Für und Wider ab: die allseits verbreitete, Mode; die Vietnamesen zu unterstützen, und die Gräuel der Amerikaner - am Ende entscheidet er sich gegen die Rezension, wohl weil er sich überlastet fühlt und weil er nicht die Kraft oder den Mut aufbringen kann bzw. will, Stellung zu beziehen. Passenderweise wird er bereits beim Abholen des Buches von einem vollen Bücherregal hinterfangen, womit auf eine letztlich zahnlose und infolgedessen verantwortungslose Bücherstubengelehrtheit verwiesen wird. Diesen Eindruck der Weltferne und einer ignoranten Gelehrtheit bekräftigen die langen Einstellungen seines Monologs, den er zu Hause im geschlossenen Raum führt." Resnais' Intention richtete sich nach eigenen Angaben allerdings darauf, dem Publikum in diesem Monolog eine „Selbstkritik des Films“ zu liefern. Ein ursprünglicher Plan zur Montage von Loin de Vietnam sah vor, den Monolog alle zehn Minuten wieder auf zunehmen. Erst bei der endgültigen Montage habe es Marker vorgezogen, die Sequenz als geschlossene zu 
Verbindung kenntlich zu machen, ist auch in Godards Film Claude Ridder kurz zu sehen (Abb.I). Eine Aufnahme von französischen Streikaktionen zeigt ihn am Rande, als Zuschauer, während der Off-Kommentar Godards die Distanz zwischen dem Intellektuellen und der Arbeiterklasse vermerkt. Dennoch geht Godard über die in Apathie mündende Skepsis des Claude Ridder hinaus, indem er eine Antwort auf das Problem anbietet und sich zugleich mit den Bedingungen filmischer Produktion und seinem eigenen Standort in diesem Bedingungsrahmen auseinandersetzt.

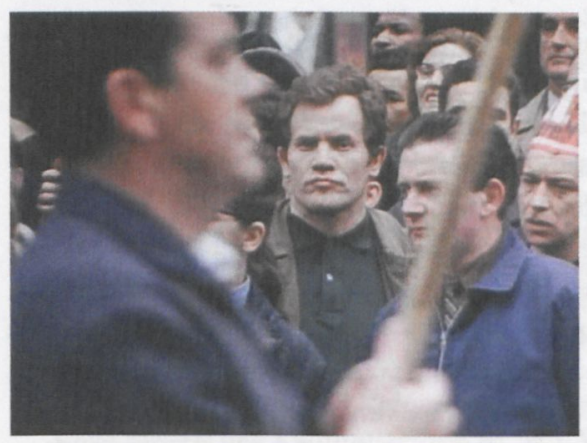

Abb. I: Der Intellektuelle und der politische Kampf. Claude Ridder (gespielt von Bernard Fresson) am Rande einer Streikaktion französischer Arbeiter in Godards Caméra-œil (1967)

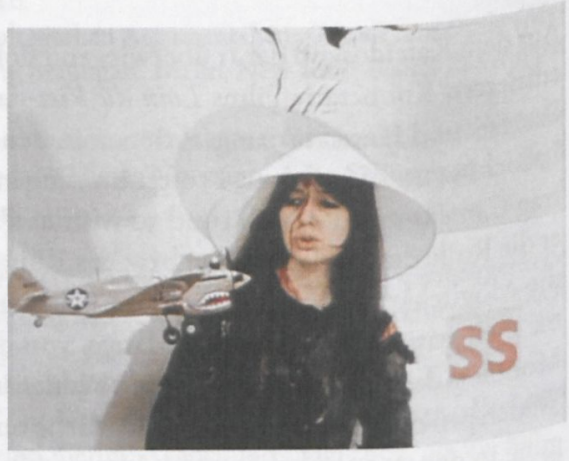

Abb. 2: Véronique (Anne Wiazemsky) als Vietnamesin im, Dialog 'mit einem amerikanischen $\mathrm{Hub}$ schrauber. Theaterszene aus La Chinoise (1967), eingeschnitten in Caméra-œil

In den folgenden Notizen möchte ich zweierlei herausarbeiten: Erstens, dass Godards Beitrag wesentlich in einer Kritik am dokumentarischen Realismus besteht, der von den anderen beteiligten Regisseuren nicht in Frage gestellt wird. Ihnen macht Godard implizit den Vorwurf, dass sie die Hollywood-Regeln zur Kontinuität filmischen Erzählens nicht außer Kraft setzen und so letztlich den US-Imperialismus bestätigen. Zweitens: Godards Solidarität mit dem vietnamesischen Volk artikuliert sich in einer radikal anderen filmischen Form, die einerseits dem Kontinuitätsprinzip des kommerziellen Spielfilms wie des klassischen Dokumentarfilms, ${ }^{4}$ auf der andern Seite aber auch dem Prinzip der Autorschaft widerspricht, das ebenfalls eine fiktionale Totalität, nämlich die souveräne kompositorische Einheitsstiftung des Regisseurs, verteidigt.' Die für Godards Filmästhetik charakteristische Dissoziation von

zeigen. Siehe Thode (wie Anm. I, S. 255)

Zum „continuity system“ des klassischen Hollywood-Films siehe David Bordwell, Janet Staiger und Kristin Thompson: The Classical Hollywood Cinema: Film Style and Mode of Production to 1960, New York: Columbia University Press, 1985. Zum narrativen Realismus auch des dokumentarischen Films siehe Henning Engelke: Dokumentarfilm und Fotografie. Bildstrategien in der englischsprachigen Ethnologic 1936-1986, Berlin 2007, u. a. S. 13.

ur politischen Bedeutung der Dissoziation von Bild und Ton und über den Zusammenhang von filmischer Kontinuitäts-Konstruktion und westlichem Identitätskonzept hat sich Godard selbst geäußert. Siehe Kent E. Carroll: Film and Revolution: Interview with the Dziga-Vertov Group, in: Focus on Godard, hg. von Royal S. Brown, Englewood Cliffs, N.J. 1972, S. 50-64, hier S. 64: "An interesting concept is your distinction between taking a picture and building a picture, and secondly, the relationship between sound and image. How 
Tönen, Bildern und Worten wird in Week-End (1967) ausdrücklich mit einem Abgesang auf die bürgerliche Kultur verbunden. Nach dem Pariser Mai 1968 sollte der Regisseur seine Abgrenzung gegen die Konventionen des kommerziellen wie des Kunst-Kinos noch verschärfen. Bis 1974 gab er die Spielfilmproduktion ganz auf, um sich gemeinsam mit Jean-Pierre Gorin einer experimentellen politischen Filmpraxis zu widmen, die keinerlei Konzession an den Zuschauergeschmack mehr zuließ und deshalb auch nicht selten ohne nennenswerte Distributionsmöglichkeiten blieb. ${ }^{6}$
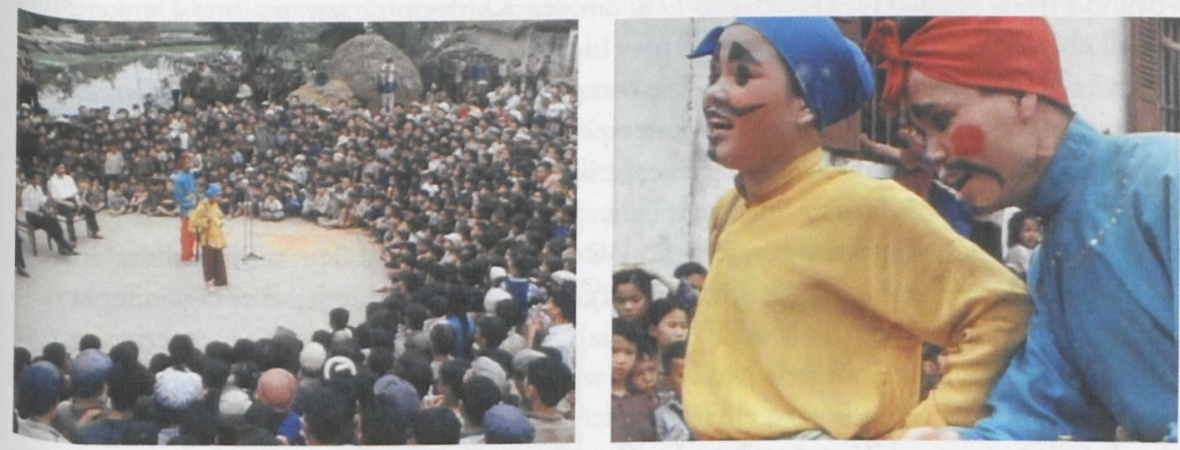

Abb. 3a, b: „Johnson weint“. Volkstheaterszene aus Joris Ivens' Beitrag zu Loin du Viet-nam, eingeschnitten in Caméra-œil

Camera-ceil ist bei aller Kürze ein Schlüsselfilm in Godards Werk, der auch als Kommentar zu $L_{a}$ Chinoise (1967) gelesen werden kann, dem ersten Spielfilm Godards, dessen Plot explizit auf die politischen Ereignisse der Zeit Bezug nimmt. Das seit $A$ bout de souffle (1959) permanent thematisierte Motiv des Ausbruchs aus gesellschaftlichen Zwängen wird hier nicht mehr direkt an die Genrelogik des Gangsterfilms, der Gesellschaftskomödie oder des Sozialdramas gebunden. Dieser Film zeigt eine ganz und gar zeitgenössische Gruppe junger Leute, die sich anders als Godards frühere Helden und Heldinnen mit ihrer gesellschaftlichen Existenz konfrontieren und mit Hilfe der Worte des großen Vorsitzenden Mao Tse-Tung den richtigen Weg zur Erkenntnis der Wirklichkeit und zur Revolution finden wollen. Veronique, gespielt von

do these concepts relate politically? / Godard: Because you belong to a certain society today in America or France, you just think that when you are speaking that your words and your structure, that they go together, that there is a complete unity. But there is no unity. There is a continuous struggle between what you say and what you think and the way we are living in a certain social condition. You are not a unity. You are trying to be a unity, but the fact is you are not. And the movie represents that in a very simple way - it's just image and sound - it's not just adding together - it's a struggle. Hollywood wants to just add them together there on the screen, just like you put a stamp on a letter. - Exactly what you mean by a film as a unity? / Godard: Well, to build it economically and aesthetically, as an ideological product for a different purpose. What is a bourgeois moviemaker doing? He is dealing with image and sound. He's building too. But for what? To achieve a truer presentation of reality, he's using hundreds of sound tracks, so that when you step on this carpet, you have the very sound of your foot on this carpet. But it means no more than that. He thinks it is real, but of course it is not. We are using the same elements, but in the way we use them we are transforming them. Our purposes are quite different.

6 Dazu Volker Pantenburg: Politik der Konfusion. Jean-Luc Godard und die Filme der Dziga Vertov-Gruppe, Kino Arsenal, Berlin, 6. Juni 2008. 
Anne Wiazemsky propagiert den Terror; Henri, gespielt von Michel Sémeniako, der sich zum Revisionismus, d. h. zur orthodoxen französischen KP bekennt, wird aus der Kommune ausgeschlossen. Guillaume, gespielt von Jean-Pierre Léaud, liebt Véronique und träumt von einem sozialistischen Theater nach chinesischem Vorbild, „das die Bücke schlägt vom, Gefängnis Kultur' zum ,Gefängnis Fabrik' [...]." 7 Er ist das alter ego des Regisseurs, der gleichwohl zu Guillaume wie zu all seinen Figuren kritische Distanz wahrt. So gleichen deren politische Äußerungen nicht selten den gestanzten Werbesprüchen, die Godard seine Protagonisten etwa in Pierrot le Fou (1965) aufsagen ließ, um den Konformitätszwang ihres Denkens und ihrer Lebensführung auszudrücken.

Einstellungen aus einer grotesken Theaterszene in La Chinoise wurden in Caméra-oeil mehrfach eingeschnitten (Abb. 2). Sie korrespondieren im Kontext des Filmganzen nun mit der dokumentarischen Aufzeichnung einer Straßentheaterszene auf einem Dorfplatz in Vietnam, die Teil von Joris Ivens' filmischem Beitrag ist (Abb. 3 a, b). Unter dem Motto ,Johnson weint' wird in dieser ersten Episode eine clowneske Parodie auf den militärischen Größenwahn der US-amerikanischen Regierung dargeboten. ${ }^{8}$ Godard teilt allerdings offenkundig nicht die Hoffnung, dass das Modell des sozialistischen Straßentheaters als Alternative zum elitären Kunstraum der bürgerlichen Kultur wirksam gemacht werden kann, indem es nach den konservativen Grundsätzen einer klassischen Filmästhetik reproduziert wird. Verfremdende Stilisierung und Zitatcharakter der Theaterszene aus La Chinoise artikulieren eine distanzierte Künstlichkeit, die von der primitivistischen Illusion des authentisch Volkstümlichen Abstand nimmt. Die wiederholt zitierte Einstellung der als Vietnamesin kostümierten Anne Wiazemsky im ,Dialog' mit einem amerikanischen Hubschrauber darf als eine bitterböse Replik auf den sentimentalen revolutionären Gestus westlicher Intellektueller gelesen werden, deren Solidarisierung mit dem vietnamesischen Volk hier als theatralisches Tableau bewertet wird. Insofern verdichtet Godard in diesem Bild gleichermaßen die Aussage von La Chinoise und von Caméra-aeil. Das politische Agieren der jungen Leute erweist sich nämlich als eine Art Ferienlager, das ein abruptes, aber ,natürliches' Ende durch die Rückkehr der Eltern Veroniques findet. Aus der Keimzelle der Revolution wird wieder die familiäre Wohnung, die rote Farbe wird entfernt. Das Politspektakel der Kommune begeistert sich an der Revolution in Südostasien, tangiert aber die heimischen Besitz- und Machtverhältnisse nicht; der vollständige Filmtitel La chinoise, ou plutôt à la chinoise enthüllt bereits diesen Als-Ob-Charakter der Handlung, die dann doch Komödie wird, also gewissermaßen die Unausweichlichkeit des Genrekino demonstriert.' Aus solcher Kritik zieht Godard, sehr viel konsequenter als die

7 Martin Schaub: Kommentierte Filmographie, in: Jean-Luc Godard. Reihe Film 19, mit Beiträgen von Francois Albera, Yaak Karsunke u. a., München / Wien 1979, S. 83-200, hier S. 155.

8 "Beginning with people gathering to watch the performance, it [das Theaterstück] shows two actors with clown-like face paint playing US president Lyndon B. Johnson and defence secretary Robert Mc Namara. Realising that they have been defeated, the actor playing Johnson sings through his tears „I have lost the war, I must go home! “ Ian Mundell: Far from Vietnam - Inside Vietnam. The genesis of the collective film Loin $d u$ Vietnam, in: European Foundation Joris Ivens Newsmagazine, Nr. 9, November 2003 , S. $25-28$, hier S. 26.

Ein Mitglied der marxistisch-leninistischen Maoisten der École normale gab seiner Empörung über G0 dards Film folgenden Ausdruck: „It's a film about bourgeois youth who have adopted a new disguise." Le Nouvel Observateur, Septembre 20, 1967. Zit. nach Richard Brody: Everything is Cinema. The Working 
anderen Filmemacher des Kollektivs, die Konsequenz, dass der Kampf des vietnamesischen Volkes „fern“ von den europäischen Gesellschaften stattfindet und von deren Angehörigen legitim nur als Anstoß zur Auseinandersetzung mit dem eigenen Metier und den ihm impliziten kapitalistischen Machtstrukturen rezipiert werden kann.

Die russische Tradition einer sozialistischen Filmästhetik ist daher eher Vorbild für Godard als die westliche Tradition des Dokumentarfilms. Der Titel Caméra-ceil zitiert den Film Kinoglaz (Filmauge, 1924) des sowjetischen Regisseurs Dziga Vertov, dem Godard und Gorin in ihrer ein Jahr später gegründeten, Groupe Dziga Vertov' explizit huldigen sollten. Vertovs dokumentarische Filmästhetik hob sich sowohl vom Realismus-Konzept nicht-fiktionaler Filme als auch vom avantgardistischen Formalismus eines Walther Ruttmann (Berlin - Die Sinfonie der Großstadt, 1929) ab. Wie Siegfried Kracauer beobachtet hat, gewann Vertov „durch die Montage dem Zusammenhang der Wirklichkeitssplitter einen Sinn ab“, wo jener nur ein

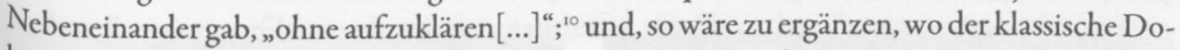
kumentarfilmer Robert J. Flaherty (Nanuk - Der Eskimo, 1922) seine gestalterischen Eingriffe verbarg, um vermeintlich das Protokoll der Wirklichkeit zu liefern.
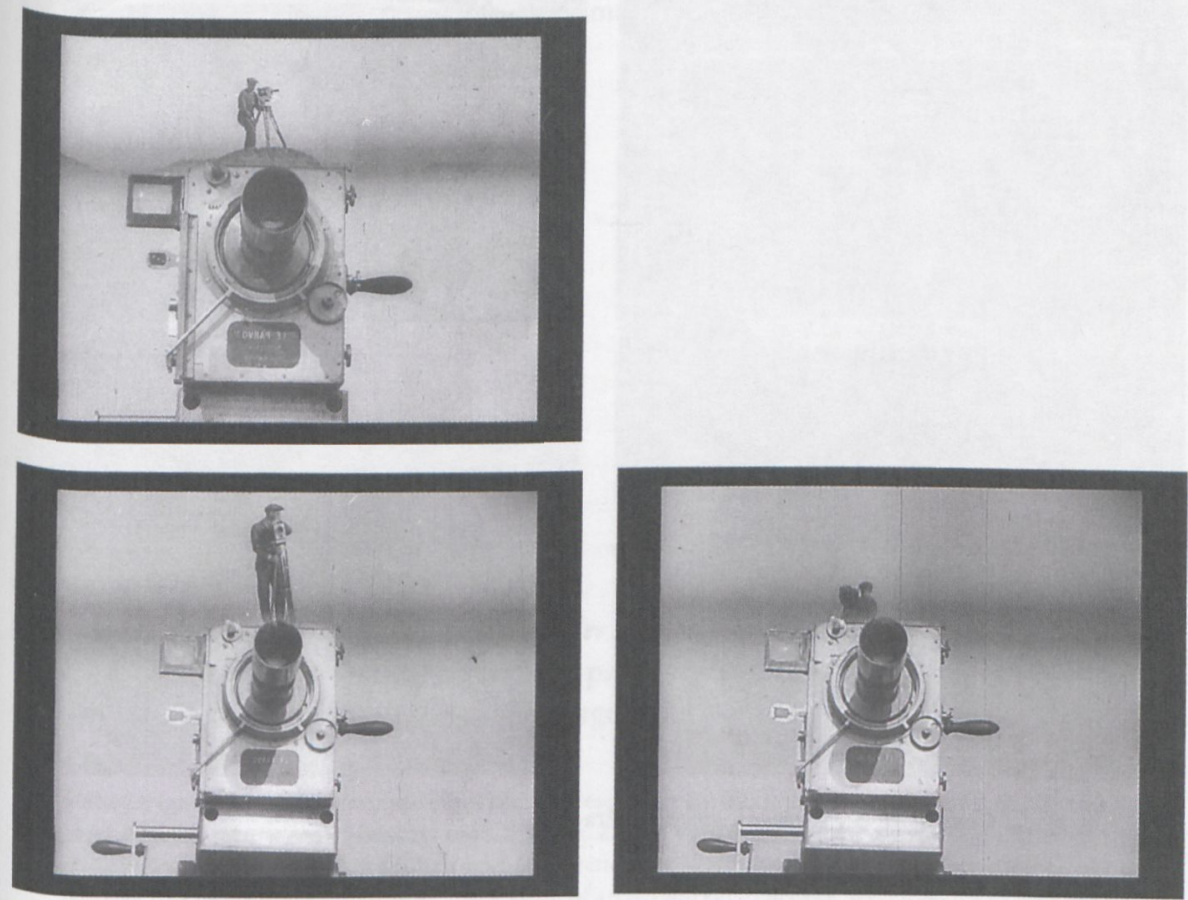

Abb. 4: a, b, c Prolog zu Der Mann mit der Kamera (Dziga Vertov, 1929) 
Das zitierte Lob Kracauers galt Vertovs Tschelowek skinoapparatom. Kinfeljeton (Der Mann mit der Kamera. Ein Filmfeuilleton, 1929). Dieser Film war offenkundig auch für Godard, der die ein Jahr später begründete Kooperation mit Gorin nicht von ungefähr nach Vertov benannte von größter Bedeutung; Vertovs während des Films wiederkehrende Selbstdarstellung als ,Mann mit der Kamera' (Abb. 4 a-c) ist unübersehbar das Modell für seinen Kurzfilm Caméra- oeil. Wie der russische Regisseur führt auch er sich und seine Kamera gleich zu Anfang ein (Abb. 5 a-d) und wiederholt wie jener während des gesamten Films immer wieder dieses Selbstporträt aus verschiedenen Perspektiven. Wenn er an den ,Lenkrädern' der schweren 35 mm Mitchell-Kamera dreht (Abb. 6), scheint er gleichsam an die von Vertov exzessiv bediente Handkurbel der Stummfilmzeit erinnern und somit jenen Transportmechanismus deutlich machen zu wollen, den James Monaco als das „Herz des Kinos“ bezeichnet hat, „denn er pumpt den Film durch die Kamera oder den Projektor. ${ }^{{ }^{\prime \prime}}$
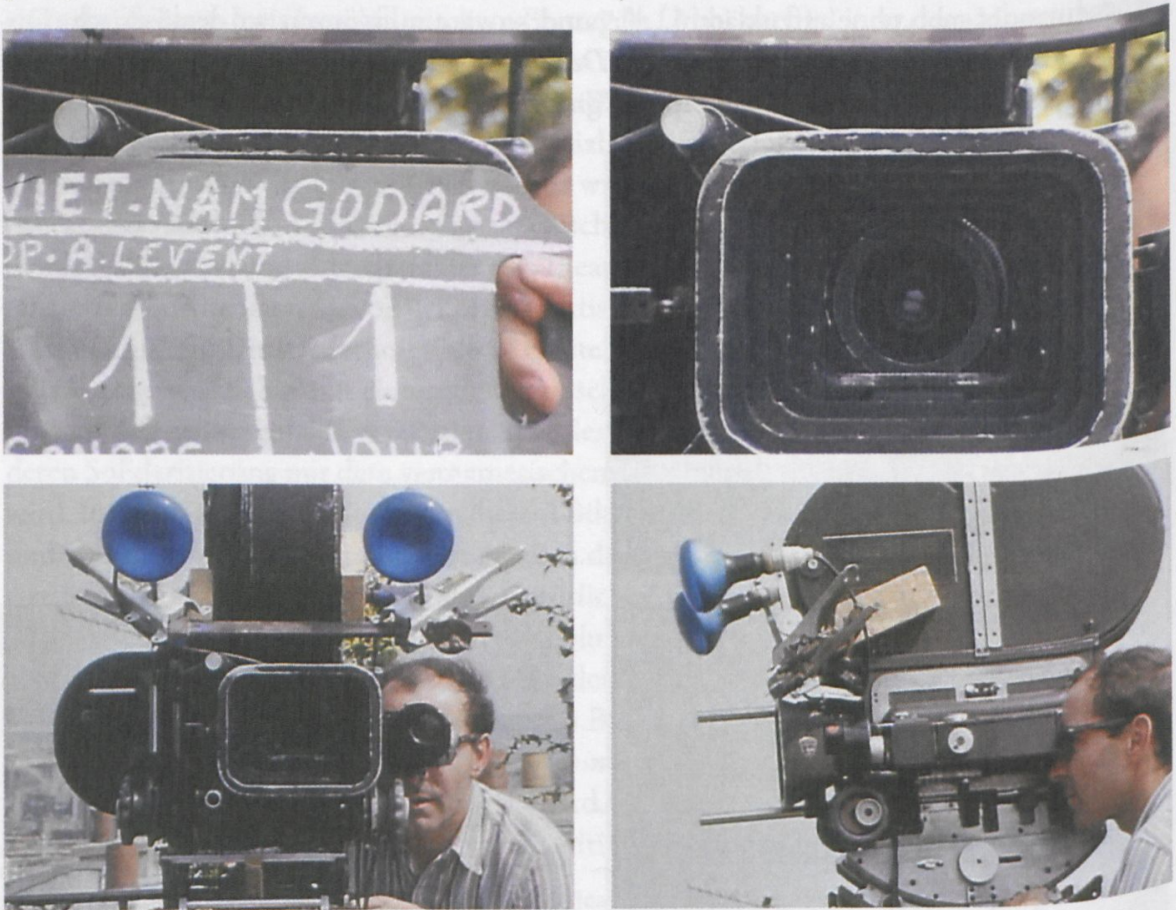

Abb. s: $a, b, c, d$ Godard an der Kamera, aus: Caméra-œil

Ebenso intensiv wie die Nachahmung von Vertovs Selbstpositionierung als Mann mit der Kamera ist freilich die in ihr manifeste Differenz. Godard teilt nicht die Emphase, mit der Vertov das Ich mit dem mechanischen Auge der Kamera gleichsetzte (Abb. 7), um mit diesem,

II James Monaco: Film verstehen. Kunst, Technik, Sprache, Geschichte und Theorie des Films und der Medien. Mit einer Einführung in Multimedia, Reinbek bei Hamburg 1980, S. 88 
Von aller Schwerfälligkeit befreiten Maschinenblick den ,neuen Menschen' zu zeigen und so auch hervorzubringen. ${ }^{12}$ Wo Vertov den Apparat als Echtheitsbeweis für seine Dechiffrierung und utopische Steigerung der vorgefundenen Wirklichkeit der Werktätigen wirksam macht, verselbständigt Godard den Verweis auf die Apparatur. In der ersten Einstellung füllt das Kamera-Objektiv zunächst den gesamten Kader und nimmt auch im sich weitenden Raum noch Zwei Drittel des Bildes ein, so dass Godard, durch den Sucher blickend, nahezu hinter der Apparatur verschwindet (Abb. s b-d). Kein offener Raum ist wahrzunehmen, die Sehmaschine verstellt den Blick. Wohl um dieser ideologiekritischen Aussage willen wählt Godard eine amerikanische Studiokamera. Unter Verzicht auf jedwede eigene ,authentische' Aufnahme definiert er den Standort des Filmautors allein über die Interpretation von Vertovs Dokumentarfilm zum neuen Rußland einerseits und über bereits vorhandenes Filmmaterial zu Vietnam andererseits. Schon seine Modifikation von Vertovs Filmprolog besagt, dass das Erbe des russischen Revolutionsfilms nicht direkt anzutreten, ein filmischer Zugang zur revolutionären Situation in Vietnam nicht möglich sei. Die von Vertov wie von Godard monumental ins Bild gesetzte Kamera wird bei aller politischen Affinität der Regisseure mit einer differenten Bedeutung versehen, wie der Vergleich der Filmanfänge deutlich machen wird.

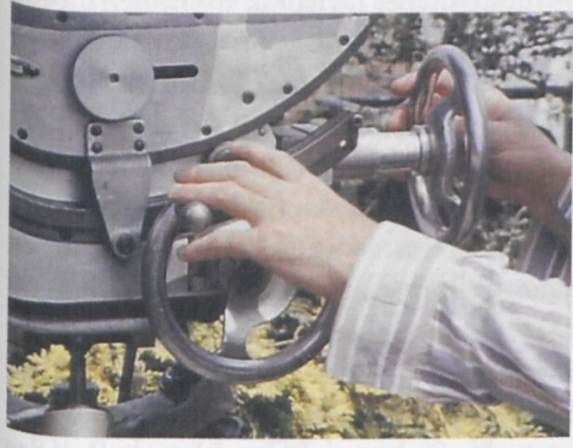

Abb. 6: Godard,kurbelt: Aus: Caméra-œil

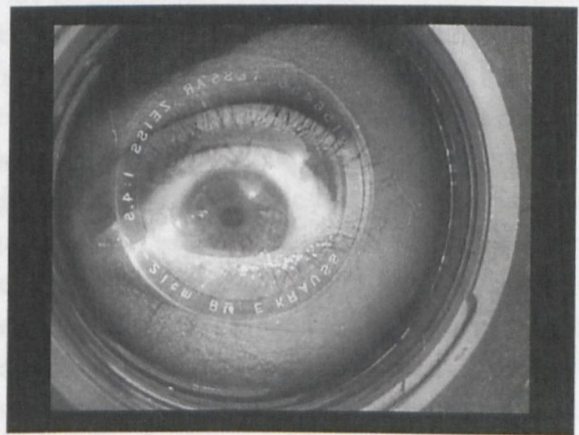

Abb. 7: Das Kino-Auge, aus: Der Mann mit der Kamera (Dziga Vertov, 1929)

Die erste Einstellung von Vertovs Mann mit der Kamera zeigt aus leichter Untersicht die frontale Ansicht der Kamera, deren oberer Rand parallel zum fernen Horizont einer Landschaft erscheint. Ihr Gehäuse wird so mit einem Bergesgipfel scheinbar eins, den Vertov ,en minia-

I2 Siehe Dziga Vertov: Wir. Variante eines Manifestes (1922), in: Texte zur Theorie des Films, hg. von FranzJosef Albersmeier, 3. Aufl. Stuttgart 1998, S. 31-35. Zu der Vertovs Mann mit der Kamera inhärenten Spannung zwischen rhythmischer Einheit und selbstreflexiver Montage vgl. John MacKay: On the one hand, we have this interest in a "rhythmic" unification of artist-worker-machine, which seems to lead to an effort to change the senses immediately, through a kind of sensory pedagogy, almost in Eisenstein's manner. [...] On the other, however, we have the whole dimension of self-reflexivity, "intellectual montage," and the use of film as a way of investigating process, construction, and the dependence of any conception of reality upon an ongoing work of representation." (Rhythm Machines: John MacKay on Dziga Vertov by Idiom December 7, 2010 (http://idiommag.com/2010/12/rhythm-machines-john-mackay-on-dzigavertov/ Letzter Zugriff 7.6.12) 
ture', die Kamera schulternd, nun von hinten erklimmt. Oben angelangt, baut er das Stativ auf, betätigt die Handkurbel der Kamera und tritt nach hinten wieder ab. Zwischengeschnitten ist die Aufnahme eines repräsentativ geschmückten Gebäudes vor ziehenden Wolken, wohl eines Theaters oder einer Akademie, deren obsoleten Status die ,Architektur' der Kamera einzunehmen beansprucht, sinnfälligerweise vielleicht auch dadurch, dass nach dem Schnitt nur noch die monumentale Kamera das Plateau für die Filmproduktion liefert, die Berglandschaft bis auf einen diffusen Horizontstreifen verschwunden ist. Man gewinnt auf diese Weise den Eindruck, dass Vertov beim Abgangvon dieser Bühne in seiner Kamera verschwindet (Abb. $4^{a-c}$ ). Er bedeutet uns, dass seine Filmkunst eine ausschließlich ökonomisch-technologische Basis hat. Der Blick vom Gipfel als Metapher des wahrheitssuchenden Kino-Auges ist nicht einem weltentrückten Genie eigen, sondern Teil und Resultat moderner Technik und industrieller Massenproduktion. Der ,rasende Filmreporter Vertov erscheint während des gesamten Films als Zeuge ebenso wie als Teil der Masse; Kameratechnik, Schnitt und Kinovorführung werden den Abläufen der Fabrikproduktion zugeordnet und als Elemente der Massenkommunikation (Telegrafie, Telefon, Plakatanschläge) und des Verkehrswesens (Eisenbahn, Straßenbahn, Flugzeug) anschaulich. ${ }^{13}$

In einem bewusst starken Gegensatz zu Vertovs futuristischer Dynamik erscheinen die formatfüllende Statik und monotone Wiederholung von Godards Selbstporträt an der schwerfälligen Mitchell-Kamera (Abb. s b, c, d). Seine verschärfte Strategie der Selbstreflexion legt implizit offen, dass Vertov in seiner visuellen Verdopplung der Kamera, die ihn als den in Aktion gesetzten Basisapparat - als das exemplarische Arbeitersubjekt - legitimiert, eine positive Idee des Absoluten voraussetzt, die im Jahr 1967 nicht mehr möglich ist. Die Einsicht in die Bedingheit der filmischen Produktion kann nicht mehr in einen sozialistischen Auftrag umgedeutet werden, sondern nur in das Zeigen der Bedingtheit münden. Anders als Vertov macht Godard deutlich, dass er als, Mann mit der Kamera' von einer anderen Kamera aufgenommen worden ist. Mit der Klappe zur ersten Einstellung (Abb. 5 a), die auf der Tonspur als solche benannt wird, lässt er demonstrativ die Spur des filmischen Produktionsprozesses stehen.

Die im engen Ausschnitt zunächst frontal, dann im Profil gezeigte Kamera konstituiert den Gegenstand des Films und die Stellungnahme Godards zu Vietnam. Immer wieder erscheint während des 15-minütigen Films, der durchaus auch (von andern Regisseuren stammende) Dokumentarbilder aus Vietnam enthält, das Bild des Regisseurs an der Kamera, positioniert in einer Pariser Dachlandschaft, wie er die Mechanik bedient und dabei monologisiert, ats ${ }^{-}$ drücklich Hand- und Kopfarbeiter zugleich. Seine Rede schließt die disparaten Bilder und Sequenzen zusammen, doch nur vermeintlich liefert sie einen Off-Kommentar im Stile der klassischen Dokumentarfilm-Ästhetik, wie ihn die erste Episode des Kollektiv-Films mit den schon erwähnten Vietnam-Aufnahmen Joris Ivens' einsetzt. Dort wird erklärt und bewertet, was zu sehen ist; die Bilder, so grausam, grotesk und anrührend sie erscheinen, sind klar einer diskursiven Logik unterworfen. Auch wenn streckenweise auf einen Off-Kommentar verzich- 
tet wird und allein mit Originalton und -geräuschen gearbeitet wird, bleibt die Transparenzillusion, mit anderen Worten - der Schein eines apparatlosen, der Wahrnehmung unmittelbar zugänglichen Geschehens - erhalten. In der Ablehnung dieser dokumentarischen Totalitätsfiktion bleibt Godard Dziga Vertov verpflichtet. Besitzt die Kamera bei Vertov jedoch noch einen ungetrübten Heldenstatus als Stellvertreterin des revolutionären Subjekts, verkörpert Godards amerikanische Studio-Kamera die Enteignung des Blicks, der nicht allein durch die visuelle Montage, sondern nur mithilfe der Rede des filmischen Autors und ihrer Verneinungskraft aufgedeckt werden kann.

Verfolgt man die Bild-Ton-Kombination in Godards Beitrag, wird man finden, dass er jede eindeutige Beziehung zwischen Bild und Ton unterläuft und mit der Monumentalisierung der Kamera die Kino-Ideologie des apparatfreien Sehens torpediert. ${ }^{14}$ Sein Monolog beginnt mit einer Erzählung über Geschehnisse während oder vor einem Bombardement. ${ }^{\text {is }} \mathrm{Zu}$ sehen sind dabei nur die Kamera und der Regisseur, was zunächst den Eindruck erzeugt, Godard filme in diesem Augenblick jenes Geschehen, von dem sein Bericht handelt. Doch die auditiv und visuell suggerierte Augenzeugenschaft wird sogleich dementiert, denn nun wechselt der Monolog vom vermeintlichen Erlebnisbericht zu kritischen Überlegungen des Regisseurs, die deutlich machen, dass er sich nicht in Vietnam befindet. Seine Rede wechselt unvermittelt von der Ebene des Augenzeugenberichts zur bildkritischen Reflexion. Vielleicht, so Godards Stimme aus dem Off, hätte er diese Szene aufgenommen, wenn er ein amerikanischer Wochenschau- oder russischer Tagesschaureporter wäre. Dokumentarische Bilder aus Vietnam - eine Schulklasse, Schützengräben - erscheinen, während der Regisseur davon berichtet, dass sein Gesuch in Vietnam zu drehen, von Hanoi abgelehnt worden sei, weil man seiner politischen Einstellung nicht traute. Es wäre jedoch ein Mißverständnis, wollte man Godards Verzicht auf die "filmische Vermittlung sichtbarer Ereignisse ${ }^{{ }_{16} 6}$ im Sinne Vertovs als Pragmatismus angesichts der ausgebliebenen Dreherlaubnis interpretieren. Godard verweist auf diese Zensurmaßnahme, um anzudeuten, dass ihr der zuvor ins Spiel gebrachte Maßstab einer professionellen Wochenschau-Ästhetik zugrundelag, deren Normen er sich nicht gebeugt hätte. Die Rede aus dem Off dient dazu, den parallel gezeigten Bildern aus Vietnam das Echtheitszertifikat zu entziehen. Im Dissens zwischen Wort und Bild entfaltet Godard die Unmöglichkeit einer audiovisuellen Annäherung an Vietnam. Während er von seinem verworfenen Plan berichtet, am Körper einer nackten Frau, „das gleichzeitig Wärmste und Lebendigste, was es gibt“, zu demonstrieren, „was ein Schrapnell aus einem Frauenkörper machen könnte“ ${ }^{17}$ sieht

I4 Vgl. Kozloff $1967 / 68$ (wie Anm.3), S. 30 : „For his part, Godard, the man who earlier castigated the right as stupid (because mean), and the left as lost (because sentimental), glues himself to a camera which despite its ominously blinking strobe lights, is so closed-framed as possibly to be photographing nothing."

Is "Mit rauher Stimme las er die Anklageschrift gegen die Frau vor. Der Mann war klein, er trug eine verwaschene graue Uniform und ging vor ihr auf und ab. Kurz darauf begannen zwei, Thunderthiefs' über uns zu kreisen, man hörte sie heulen, als sie zum Tiefflug ansetzten. Und man hörte die Detonation ihrer Bomben [...]. Zit. nach dem Abdruck des Monologs in der Frankfurter Rundschau vom 19.10. 1967. Hieraus auch die folgenden Zitate im Text.

I6 So Vertovs Information zum Film im Vorspann zu Der Mann mit der Kamera.

17 Godards ursprünglicher Beitrag bestand aus der Aufnahme einer nackten Frau, begleitet von einem Kommentar, der die Auswirkung eines solchen Bombenangriffs auf ihren Körper beschrieb. Er wurde von der Gruppe zurückgewiesen, die auch seinen in letzter Minute gelieferten Beitrag Caméra æil nur zögernd 
man nichts als seine Hand an den Steuerungselementen der Kamera, die Schwenks ausfuihrt (Abb. 6). „Es scheint mir schwierig, über Bomben zu reden, wenn sie einem nicht selbst auf den Kopf fallen", fährt er fort. Und während unscharfe Aufnahmen von entlaubten Wäldern und vergifteten Fischen gezeigt werden, stellt der Off-Kommentar die Frage, was die angemessene Form für diesen katastrophischen Gehalt sei.
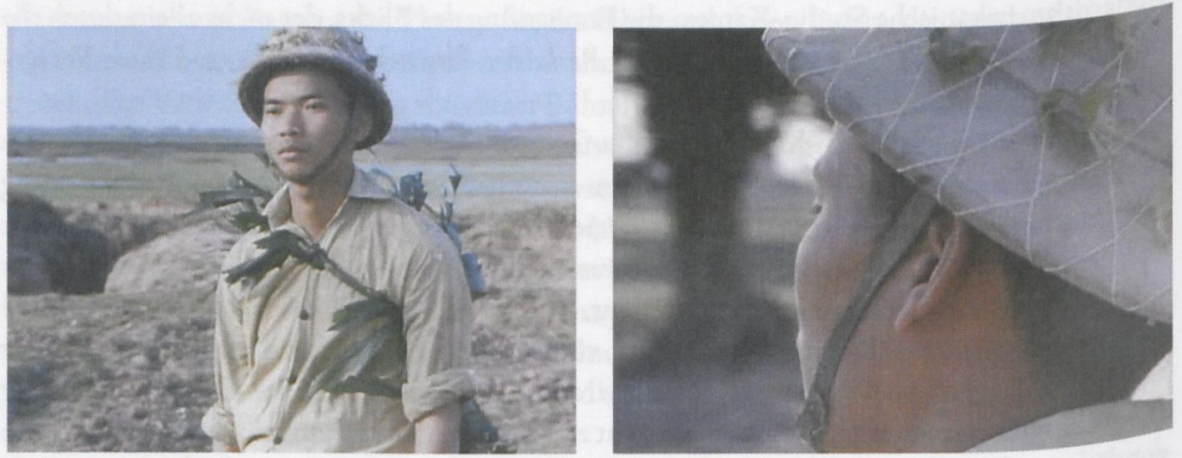

Abb. 8: a, b, Vietnam als,Symbol des Widerstands', aus: Caméra-œil

Godards Vorbehalt gilt dem bürgerlichen Modell ästhetischer Erfahrung. Gegen die üblicherweise eingesetzte manipulative Emotionalisierung der Zuschauerrezeption stellt er die Reflexion auf den eigenen gesellschaftlichen Standort: „Das Beste, was ich machen kann für Vietnam, ist, statt es mit meiner Hochherzigkeit zu überschütten, mich von ihm ergreifen zu lassen, mir klarzumachen, welchen Platz es in unserem Leben einnimmt, überall." Vietnam wird zu einem „Symbol des Widerstands“ erklärt, das jedem in seiner eigenen Existenz auferlege, Stellung zu beziehen. Während die Kamera die Gestalt eines jungen vietnamesischen Kämpfers umfährt und gleichsam zum Denkmal monumentalisiert (Abb. $8 \mathrm{a}, \mathrm{b}$ ), spricht Godard aus dem Off von der notwendigen Aneignung seines Widerstands. „Wenn man in Guinea ist, muß man gegen die Portugiesen sein, in Chicago für die Schwarzen [...]." Sein eigener Kampf gilt dem Widerstand gegen den ,wirtschaftlichen und ästhetischen Imperialismus des amerikanischen Kinos, das inzwischen das Kino der ganzen Welt zerrüttet" habe. Diesen Kampf sieht er gleichgerichtet mit dem der streikenden Arbeiter bei der Rhodiacéta in Besançon, ${ }^{18}$ während eine reale Beziehung zur Arbeiterschaft nicht möglich sei: „Das Arbeiterpublikum schaut sich meine Filme nicht an und zwischen mir und ihm gibt es die gleiche Trennungslinie wie zwischen mir und Vietnam oder ihm und Vietnam." Schließlich zitiert Godard den Surrealisten André Breton: „Ich glaube an die absolute Kraft all dessen, was, spontan oder nicht, getan wird, das Einverständnis zu verweigern." Es gehe darum, den Schrei weiterzuleiten, den „uns in jedem Augenblick das erschreckende Missverhältnis zwischen dem Gewonnenen und dem Verpassten, dem Zugestandenen und dem Erlittenen abnötigt." Godard stellt also mit Breton dem

akzeptierte. Brody 2008 (wie Anm. 9), S. 3 Ir. Allerdings kritisiert auch Godard selbst in Caméra- ceil seine erste Idee als etwas „ästhetisch Gesuchtes“. 
absoluten, der Welt zugewandten Kino-Auge Vertovs die Absolutheit der Negation entgegen. Sein Film ist jedoch keineswegs auf eine solche eher romantisch anmutende anarchistische Position zu reduzieren, so wenig wie der Monolog Godards schon den Inhalt des Films ergibt.

Obwohl Caméra-ceil als Monolog angelegt scheint und oft allein durch dessen Referierung beschrieben wurde, erschließt der Text allein den Film nicht, macht er etwa nicht verständlich, warum sich der Regisseur mit einer amerikanischen Studiokamera, dem Gerät der feindlichen imperialistischen Macht darstellt, warum er tote Fische und - dann doch - verstümmelte Leichen zeigt, obgleich er ausdrücklich bekennt, in Paris arbeiten zu wollen, warum er die politischen Theaterszenen aus La Chinoise und Aufnahmen von Claude Ridder in seinen Filmbeitrag aufnimmt. Durch diese Verwendung schon vorhandener Bilder nimmt Godard von einem traditionellen Konzept der Autorschaft Abstand, ebenso - paradoxerweise - in seinem Selbstporträt. Denn er führt sich quasi als Anhängsel der Kamera-Maschinerie ein (Abb. 5 a-d), als jemand, der notwendig dem Diktat Hollywoods unterworfen bleibt, verschmolzen geradezu mit dem filmischen Apparat (Abb. 9). ${ }^{19}$

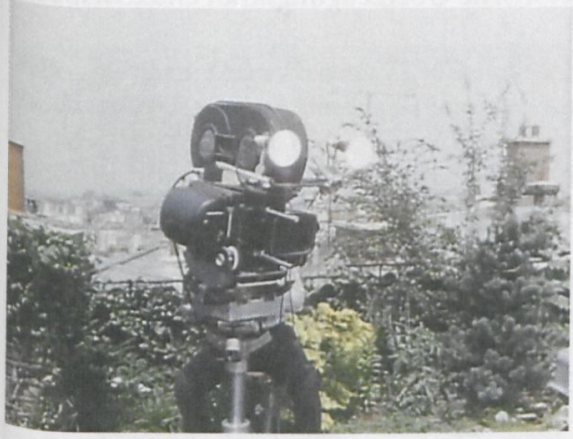

Abb. 9: Godard an der Kamera, aus: Caméra-œil

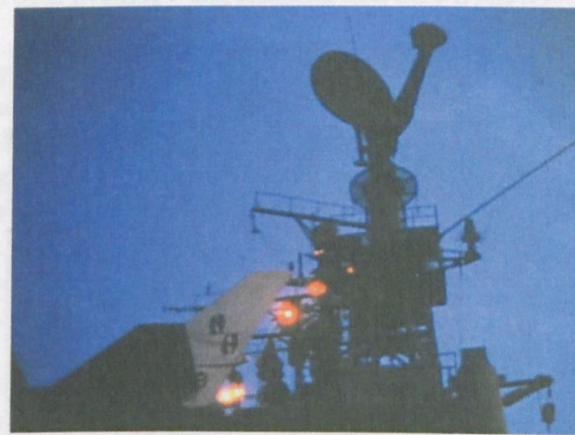

Abb. 1o: Radarturm des amerikanischen Flugzeugträgers, Kitty Hawk: Prolog zu Loin du Viet-nam.

Die gigantische Mitchell-Kamera verweist überdies auf den Prolog des Gesamtfilms, der den Radarturm des amerikanischen Flugzeugträgers ,Kitty Hawk' und die Beladung mit Bomben zeigt (Abb. IO, II). Die Bewegung des Ortens zum Zweck militärischer Aktion ist hier nun gleichsam dem Sucher der Kamera übertragen (Abb. 12). Das Kameraobjektiv wiederum korrespondiert einem Geschosslauf. Es ist, im Vorgriff auf die Ideologiekritik der sog. Apparatus-Debatte, ${ }^{20}$ als Herrschaft sichernde Waffe visualisiert. Auch auf der filmhistorischen Zitatebene ist dieser Vergleich unübersehbar: Der,Schuss' aus dem Bild ruft das revolutionäre

I9 Bereits im Prolog zu Le Mépris (1963) erscheint die schwere Mitchell-Kamera als Apparat der amerikanischen Filmindustrie. Ihre diegetische Verwendung - Gegenstand des Films ist die Produktion eines Films über die Odyssee - wird ergänzt durch einen außerdiegetischen Verweis auf Godards Filmproduktion selbst, denn die Kamera bedient Raoul Coutard. Zur selbstreflexiven Dimension dieses Films siehe Verf.: "Comme au cinéma. Le ciel est bleu." Zur ästhetischen Tradition der Himmelsschau und ihrer Bedeutung im Frühwerk Jean-Luc Godards, in: Figurationen 2/2010, hg. von Hans Georg von Arburg, S. 39-67.

20 Dazu Robert F. Riesinger (Hg.): Der kinematographische Apparat. Geschichte und Gegenwart einer interdisziplinären Debatte. Unter Mitwirkung von Guntram Geser, Münster 2003. 
Kanonenfeuer des Panzerkreuzers Potemkin in Erinnerung, ebenso den dadaistischen Schuss ins Publikum aus dem Prolog von René Clairs Entr'Acte (1924). Godard setzt das Motiv jedoch grundsätzlich sehr viel deutlicher als metafilmisches Bild ein. ${ }^{21}$ In Caméra-aeil gibt er der Schuss-Metapher eine radikal politische Deutung. Die Bilder des Regisseurs an der Kamera, als fast chaplinesker Arbeiter an der Maschine und partiell mit ihr zu einem roboterartigen Wesen verschmelzend (Abb. 9), in der Profileinstellung aber auch immer wieder zum individuellen Beobachter und Sprecher zusammengesetzt (Abb. sd), sind, in der Verschränkung mit den Vietnambildern und dem Off-Monolog Godards, symbolisch hochverdichtet, was jene Kritiker, die dem Selbstporträt Nichtigkeit und Leere vorwarfen, ${ }^{22}$ nicht realisierten. Godards Kampf gegen den amerikanischen Kino-Imperialismus gilt letztlich der Konzeption des Subjekts, das seinen Identitätsentwurf den Helden Hollywoods entlehnt. ${ }^{23}$ Den falschen, durch die filmische Raumkonstruktion gestifteten Einheitsentwurf zerstört Godard, indem er Bild und Ton in eine diskontinuierliche Beziehung bringt. Um den Filmapparat zu einer Waffe für einen gerechten Krieg um zu schmieden, musste er die Fiktionalität des Dokumentarischen aufweisen und den Bildern aus Vietnam ihre rhetorische Überzeugungskraft entziehen.

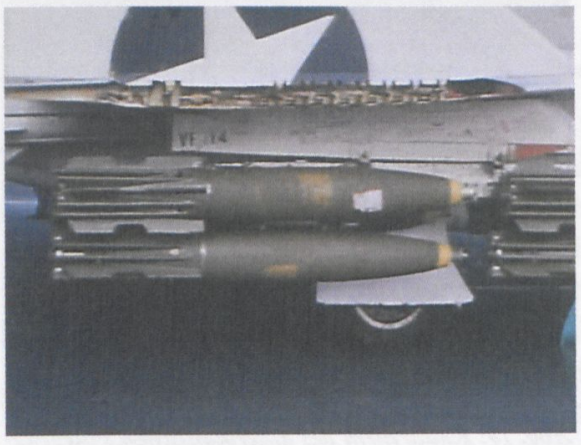

Abb. Ir: Beladung eines Flugzeugs mit Bomben. Pro$\log z u$ Loin du Viet-nam

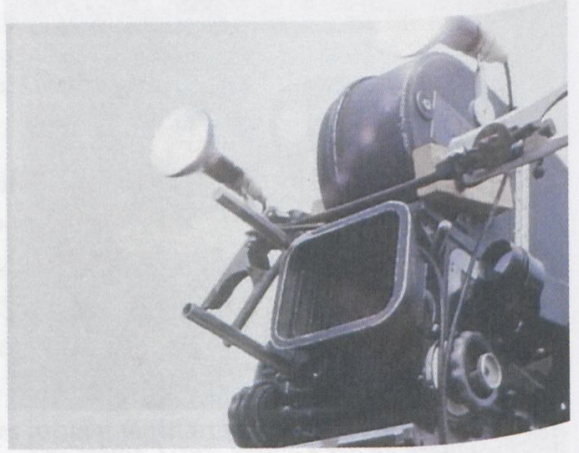

Abb. 12: Die Kamera visiert ihr Ziel an, aus: Caméra-œil

\section{Abbildungen}

Abb. I: Der Intellektuelle und der politische Kampf. Claude Ridder (gespielt von Bernard Fresson) am Rande einer Streikaktion französischer Arbeiter in Godards Caméra-eil (1967)

Zu Godards Vergleich von Kamera und Schusswaffe siehe Regine Prange: Genre und Genrekritik. Der Western in Jean-Luc Godards À bout de souffle (1959). In: Ursula Frohne und Lilian Haberer (Hg.): Kinematographische Räume, München 2012, S. 621-66o.

Z. B. Raymond Durgnat: Far from Vietnam, in: Films and Filming, Vol. 14, no.5, Febr. 1968, S. 2 of., hier S. 2r. Zu den Schwachstellen des Films gehöre „Godard's criticism-defying, but empty, device of having himself photographed looking through a big Mitchell camera [...]."

23 Auf derselben Ebene liegt seine Problematisierung des politischen Engagements von Jane Fonda in Letter to Jane von 1972 , den Godard gemeinsam mit Jean-Pierre Gorin drehte. Hinweis Ralf M. Fischer. 
Abb. 2: Véronique (Anne Wiazemsky) als Vietnamesin im ,Dialog' mit einem amerikanischen

Hubschrauber. Theaterszene aus La Chinoise (1967), eingeschnitten in Caméra-ail

Abb. 3: a,b „Johnson weint“. Volkstheaterszene aus Joris Ivens' Beitrag zu Loin du Viet-nam, eingeschnitten in Caméra-ceil

Abb. 4: a, b, c Prolog zu Der Mann mit der Kamera (Dziga Vertov, 1929)

Abb. s: a, b, c, d Godard an der Kamera, aus: Caméra-aeil

Abb. 6: Godard ,kurbelt'. Aus: Caméra-aeil

Abb. 7: Das Kino-Auge, aus: Der Mann mit der Kamera (Dziga Vertov, 1929)

Abb. 8: Vietnam als, Symbol des Widerstands', aus: Caméra-ceil

Abb. 9: Godard an der Kamera, aus: Caméra-ceil

Abb. 10: Radarturm des amerikanischen Flugzeugträgers, Kitty Hawk'. Prolog zu Loin du Viet-nam.

Abb. Ir: Beladung eines Flugzeugs mit Bomben. Prolog zu Loin du Viet-nam.

Abb. 12: Die Kamera visiert ihr Ziel an, aus: Caméra-ceil

\section{Literatur}

Beller, Jonathan: Dziga Vertov \& The Film of Money, in: Boundary 2 26.3, (1999) I5I-199

Bordwell, David; Staiger, Janet und Thompson, Kristin: The Classical Hollywood Cinema: Film Style and Mode of Production to 1960 , New York: Columbia University Press, 1985

Brody, Richard: Everything is Cinema. The Working Life of Jean-Luc Godard, London 2008

Carroll, Kent E.: Film and Revolution: Interview with the Dziga-Vertov Group, in: Focus on Godard, hg. von Royal S. Brown, Englewood Cliffs, N.J. 1972, S. 50-64

Durgnat, Raymond: Far from Vietnam, in: Films and Filming, Vol. 14, no.5, Febr. 1968, S. $20 f$.

Engelke, Henning: Dokumentarfilm und Fotografie. Bildstrategien in der englischsprachigen Ethnologie 1936-1986, Berlin 2007

Idiom: Rhythm Machines: John MacKay on Dziga Vertov, December 7, 2010 (http://idiommag. $\mathrm{com} / 2010 / 12 /$ rhythm-machines-john-mackay-on-dziga-vertov/

Kozloff, Max: Shooting at Wars, in: Film Quarterly, Vol. 21, no.2, Winter 1967/68, S. 27-31

Monaco, James: Film verstehen. Kunst, Technik, Sprache, Geschichte und Theorie des Films und der Medien. Mit einer Einführung in Multimedia, Reinbek bei Hamburg 1980

Mundell, Ian: Far from Vietnam - Inside Vietnam. The genesis of the collective film Loin du Vietnam, in: European Foundation Joris Ivens Newsmagazine, Nr. 9, November 2003, S. $25-28$

Pantenburg, Volker: Politik der Konfusion. Jean-Luc Godard und die Filme der Dziga Vertov-Gruppe, Kino Arsenal, Berlin, 6. Juni 2008

Prange, Regine: „Comme au cinéma. Le ciel est bleu.“ Zur ästhetischen Tradition der Himmelsschau und ihrer Bedeutung im Frühwerk Jean-Luc Godards, in: Figurationen 2/2010, hg. von Hans Georg von Arburg, S. 39-67

Prange, Regine: Genre und Genrekritik. Der Western in Jean-Luc Godards A bout de souffle (1959). In: Kinematographische Räume, hg. von Ursula Frohne und Lilian Haberer, München 2012, S. 621-660

Schaub, Martin: Kommentierte Filmographie, in: Jean-Luc Godard. Reihe Film 19, mit Beiträgen von Francois Albera, Yaak Karsunke u. a., München / Wien 1979, S. 83-200

Temple, Michael, James S Williams und Michael Witt (Hg.): For ever Godard, London 2007

Thode, Thomas: Loin du Viêt-nam [Fern von Vietnam], 1967, in: Chris Marker. Filmessayist, hg. von Birgit Kämper und Thomas Thode, München 1997, S. 251-255.

Vertov, Dziga: Wir. Variante eines Manifestes (1922), in: Texte zur Theorie des Films, hg. von Franz-Josef Albersmeier, 3. Aufl. Stuttgart 1998, S. 31-35 